\title{
FREQUENCY OF STELLAR COLLISIONS IN THREE-BODY HEATING
}

\author{
DAVID F. CHERNOFF AND XIAOLAN HUANG \\ Department of Astronomy \\ Cornell University \\ Ithaca, NY 14853
}

\begin{abstract}
The probability for collisional interaction of three body binaries is calculated as a function of the physical radius and mass of the stellar objects and the depth of the cluster potential well. For typical cluster parameters, there is a significant chance of physical collision for objects as small as white dwarfs. One consequence of the collisions is to lower the amount of heat produced from hardening a binary, thereby diminishing the efficiency of the three-body heating mechanism.
\end{abstract}

\section{Introduction}

When a cluster undergoes core collapse stellar interactions tend to harden the binaries and some of these encounters lead to physical collisions. In this paper, we explore the frequency of stellar collisions involving binaries formed as a result of three-body encounters (3B binaries, hereafter). Such binaries are born when the core reaches high densities, interact predominantly with stars in the core and release heat at a rate that roughly balances the transport of energy to the outer regions of the cluster by gravitational relaxation (for a review Goodman 1989). Some of the encounters eject stars and/or binaries from the cluster center; some lead to physical collisions. Generally, it has been assumed that finite size effects are unimportant for the case of 3B binary heating, an assumption explored here in some detail. By way of contrast, it is well-known that finite size effects are crucial to understanding the role of two-body binaries (binaries formed in dissipative encounters of two single stars, hereafter 2B-binaries). For example, the cross section for the formation of $2 \mathrm{~B}$ binaries is the subject of ongo- 
ing work to disentangle the events that lead to collisions and mergers from those that actually produce binaries (Kochanek 1992, Mardling 1995ab). Collisional processes will limit the ability to extract binding energy since a strong encounter with a free star often yields a complicated hydrodynamical interaction instead of a more tightly bound binary (Davies, Benz and Hills 1993, 1994). Clearly, the efficiency of cluster heating is altered by these collisional effects.

Under typical cluster conditions, newly formed 3B binaries have large semi-major axes compared to the stellar radii of main sequence or compact constituents. Let $x=E_{b} / m_{s} \sigma_{s}^{2}$ be the binary hardness, where $E_{b}$ is the binary energy, $m_{s}$ is the mass of a single star, $\sigma_{s}$ is the one-dimensional velocity dispersion of the singles and we assume that the translational degrees of freedom of binaries and singles are in thermal equilibrium. Then $x=4.4(\mathrm{AU} / a)\left(\left[m_{1} m_{2} / m_{s}\right] / M_{\odot}\right)\left(10 \mathrm{~km} / \mathrm{s} / \sigma_{s}\right)^{2}$ where $a$ is the semi-major axis and $m_{1}$ and $m_{2}$ are the mass elements of the binary. 3B binaries form at small $x$ and harden until the recoil from a strong encounter liberates them from the cluster potential. For an average energy change of $0.4 E_{b}$, ejection occurs for hardness $x_{c}=2.5\left(m_{1}+m_{2}\right)\left(m_{1}+m_{2}+m_{s}\right) \Phi(0) / m_{s}^{2} \sigma_{s}^{2}$ where $\Phi(0)$ is the depth of the potential in the core. The corresponding semi-major axis is $a_{c} / \mathrm{AU}=2.1 \times 10^{-2} y\left(10 \mathrm{~km} / \mathrm{s} / \sigma_{s}\right)^{2}\left(14 \sigma_{s}^{2} / \Phi(0)\right)$, where $y=\left(\left[6 m_{1} m_{2} m_{s} /\left(m_{1}+m_{2}\right)\left(m_{1}+m_{2}+m_{s}\right)\right] / M_{\odot}\right)$, or $a_{c}=4.6 R_{\odot}$ for the fiducial parameters. The experiments described in this paper show that $r e$ peated resonant encounters raise the probability for collision dramatically so that white-dwarf 3B binary systems will often collide before they harden enough to be ejected. (A more detailed report, Huang and Chernoff 1995, is in preparation.)

\section{Binary History}

Assume a background of single stars with the following properties: homogeneous in space, steady-state in time, Maxwellian in velocity. Place a binary in the sea of stars and follow its history, assuming that the density is low enough that all changes may be described in terms of pairwise encounters between the binary and a single star. Each interaction alters the essential parameters of the binary (the semi-major axis, the eccentricity, the orientation of the plane of the orbit, and so forth). Denote the sequence of parameter states that exist between the pairwise encounters as a "binary history."

A specific realization of a binary history involves generating the initial conditions for an encounter, integrating the three-body problem to find the final state and repeating. From the vast set of possible encounters, only those with pericenters $\leq \zeta a$ in the two-body limit are considered. Here $a$ is 
the current semi-major axis and $\zeta$ is a constant, chosen to be large enough to include all "important" encounters. The mean rate of encounters is

$$
\frac{d N}{d t}=\int d^{3} v f(v) \pi(\zeta a)^{2}\left|v-v_{B}\right|\left[1+\frac{2 G M_{t}}{\zeta a\left|v-v_{B}\right|^{2}}\right]
$$

where $M_{t}$ is the total mass of the stars involved and $v_{B}$ is the binary velocity. Specific encounters are realized as a Poisson process subject to the above mean rate. All initial conditions (impact parameter, velocity of approach at infinity, orientation angles) are drawn from the appropriate probability distributions. The outcome of each encounter is calculated numerically by a three-body integration and the endstate is one of three types: flyby, exchange or ionization. In addition, the outgoing stellar velocity, binary center of mass velocity, closest distance of approach, and other quantities are calculated. A new encounter is realized and the process repeated until (1) a collision occurs, (2) the binary escapes, or (3) the binary is ionized.

The value of $\zeta$ was fixed as follows. The rate coefficients for the average energy change, the mean square energy change, the average angular momentum change, and the mean square angular momentum change were calculated for hard binaries of a range of $x$ and for $\zeta=4,8,12,16$ and 20. The error incurred for $\zeta=12$ was less than $1 \%$ in all quantitites. In addition, the mean and mean square energy changes at selected $x$ agreed with the results of Heggie and Hut (1993) to within the statistically dominated errorbars.

\section{Steady-State}

Assuming a steady-state homogeneous, single star population, Goodman and Hut (1993, hereafter GH93) found the steady-state binary distribution $f(x)$. At small $x$ the 3B binary creation rate is high but the probability of ionization is large; at large $x$ the creation rate is small but the survival probability is high. Eventually, for $x \gtrsim x_{c}$ recoil ejects binaries from the potential well of the cluster. The solid lines in Figure 1 display $f(x)$ for three potential depths; there are many soft binaries and they are closely approximated by a thermal distribution (dotted line); the distribution function is flat at large $x$, corresponding to a constant net flux until ejection becomes possible. Binaries at large $x$ are immortal, i.e. they have virtually no chance of being ionized.

A direct computation of the steady-state distribution has been carried out as follows. A number of binary histories $(16,000)$ were created. The hardness at birth was distributed according to the $3 \mathrm{~B}$ binary creation rate but confined to the range $x>0.3$; the eccentricity distribution was thermal. (The range in $x$ accounts for roughly $40 \%$ of the immortal binaries in the 


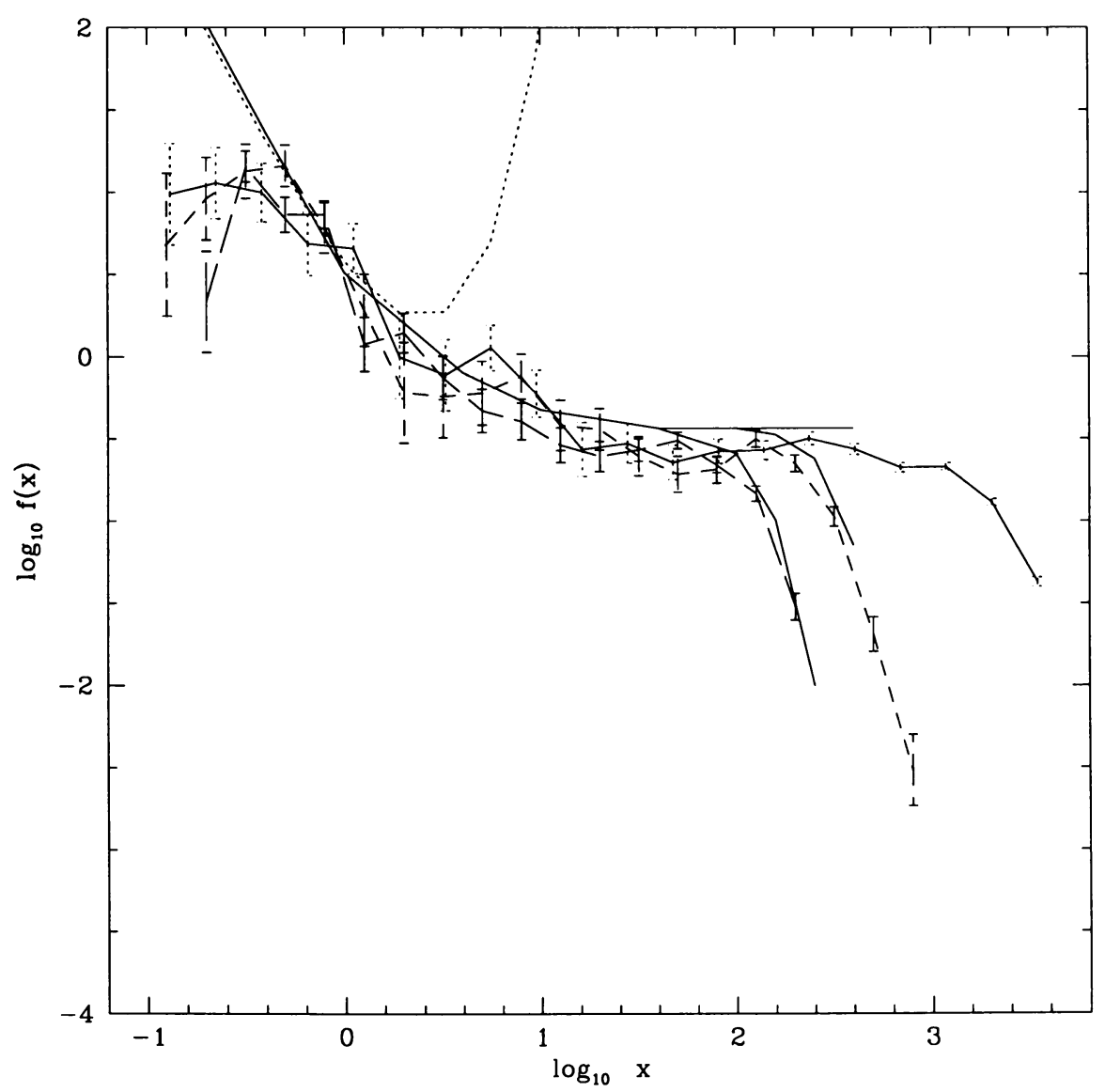

Figure 1. The solid line shows the steady-state distribution funciton found by Goodman and Hut (1993) for three different escape criteria $\left(\Phi(0) / \sigma_{s}^{2}=6,14\right.$ and $\left.\infty\right)$. The lines through points with error bars are the same results based on the binary history (long-dashed, short-dashed and solid correspond to $\Phi(0) / \sigma_{s}^{2}=6,14$ and 128 , respectively). The dotted line gives the distribution in thermal equilibrium.

GH93 solution but it is impractical to decrease the lower limit of $x$ by much, since the probability of survival for a binary with $x=0.3$ is $\sim 2 \times 10^{-3}$ and decreases rapidly. Tests briefly described below show that it is possible to "renormalize" the distribution function to include the effect of births with $x<0.3$.) The time span of the simulation was long enough to realize the steady-state binary distribution. The result is illustrated in Figure 1 (see points with errorbars) and agrees in shape quite well with GH93 for 
$x \gtrsim 1$. The discrepancy at small $x$ is a consequence of the truncated range of the birth function. The fraction of binaries which are born with a given $x$ that go on to become immortal binaries agrees well with previous analyses (Hut 1985, GH93). About 1\% of the histories yielded immortal binaries (on average, $\sim 360$ encounters per history) and the rest were destroyed ( $\sim 170$ encounters).

The results of the binary history may be used to examine how quickly memory of the initial conditions of a binary's birth are lost. For example, a population of newly formed binaries with $0.6<e<0.7$ and $1<x<2$ evolves a thermal distribution of eccentricity in the time necessary for $\sim 42$ encounters (within 12a) for a binary with $x=1$. (The number of encounters for individual members of the population ranges greatly.) Detailed analysis (see Huang and Chernoff 1995) shows that the memory of the specific initial conditions ( $x$ and eccentricity) is quickly lost so that the statistical properties of the binary population that have hardened to $x \gtrsim 3$ are nearly independent of the detailed form of the binary birth distribution. Since the issues of interest in this paper concern the binary behavior as $x$ grows large, we have simply renormalized the birth rate to yield the immortal birth rate from GH93.

\section{Close Encounters}

Consider the steady-state distribution of immortal binaries. At any instant, there will be a range of values of $x$ and range of values of $d_{\text {min }}$, the closest approach suffered by any star which is or was a member of the binary. Figure $2 \mathrm{a}$ is a realization of the steady-state distribution of the values of $\left(x, d_{\min }\right)$. The evolution of the points as they age can be understood fairly simply: the hard binaries tend to move to larger values of $x$ (but not monotonically) and the value of $d_{\min }$ always decreases. Note that the objects that eventually disrupt are not included. The general flux in Figure 2a is down and to the right. Figure $2 \mathrm{~b}$ is an analogous plot for the distribution of $\left(V_{\max }, d_{\min }\right)$ where $V_{\max }$ is the maximum recoil the binary has ever suffered; all points move monotonically down and to the right. The rate of loss by ejection is the flux across the line $V_{\max }$ equals the escape velocity. A vertical line is included in the diagram for illustrative purposes. Likewise, the collision criterion corresponds to the flux across a horizontal line at a given value of $d_{\text {min }}$.

It is striking that so many of the points in the steady-state snapshot have undergone close encounters. The collision probability for an immortal binary in a sea of similar stars may be inferred from the two dimensional distribution. Figure 3 illustrates the probability as a function of the size (in dimensionless units). For fiducial parameters of $\sigma_{s}=10 \mathrm{~km} / \mathrm{s}, r_{\min }=2 R_{*}$, 

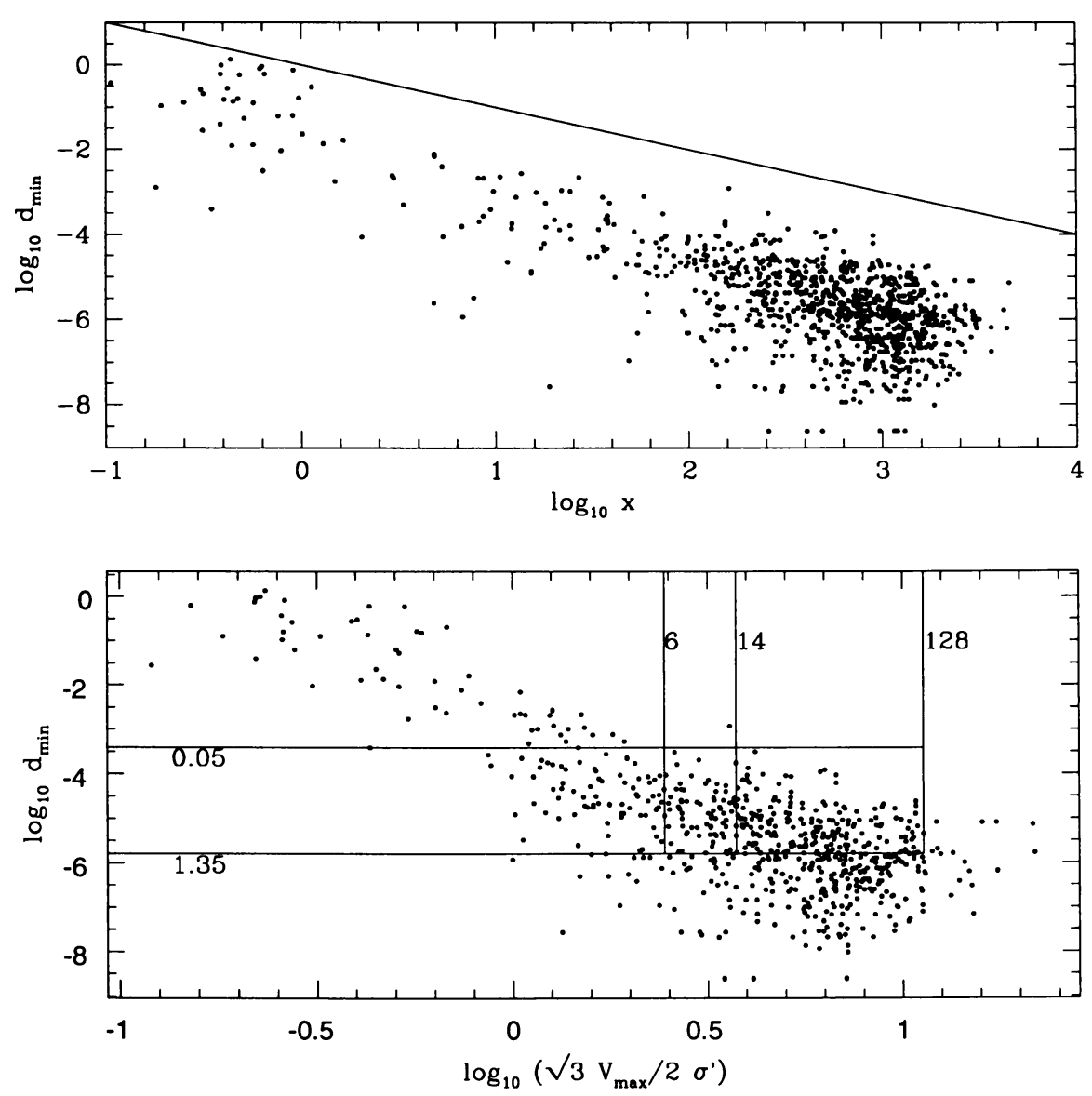

Figure 2. Top: The steady-state distribution of hardness and distance of closest approach for immortal 3B binaries ( $w i t h x=3 G m / 4 a \sigma^{\prime 2}, \sigma^{\prime 2}=\sigma_{s}^{2}+\sigma_{B}^{2}$, and $d_{\min }=4 d \sigma^{\prime 2} / 3 G m$ ). Binaries are born in range $x>0.3$. All points must lie below the solid line which is the condition $a=d_{m i n}$. Bottom: The steady-state distribution of maximum binary recoil $\left(\sqrt{3} V_{\max } / 2 \sigma^{\prime}\right)$ and distance of closest approach for immortal 3B binaries. The vertical lines mark the escape velocity in clusters with $\Phi(0) / \sigma_{s}^{2}=6,14$ and 128 ; the horizontal lines mark $d_{\min }$ for 0.05 and $1.35 M_{\odot}$ white dwarfs.

then the abscissa is marked to indicate the location of neutron stars with $1.4 M_{\odot}, R_{*}=10^{6} \mathrm{~cm}$ at -7.7 and and of main sequence stars with $1.0 M_{\odot}$, $R_{*}=R_{\odot}$ at -2.7 . In addition, white dwarfs span a range from -5.8 to -3.4 depending upon mass (heavy ones with $1.35 M_{\odot}, R_{*}=10^{-3} R_{\odot}$ lie at the small end, light ones with $0.05 M_{\odot}, R_{*}=10^{-2} R_{\odot}$ at the large end). 


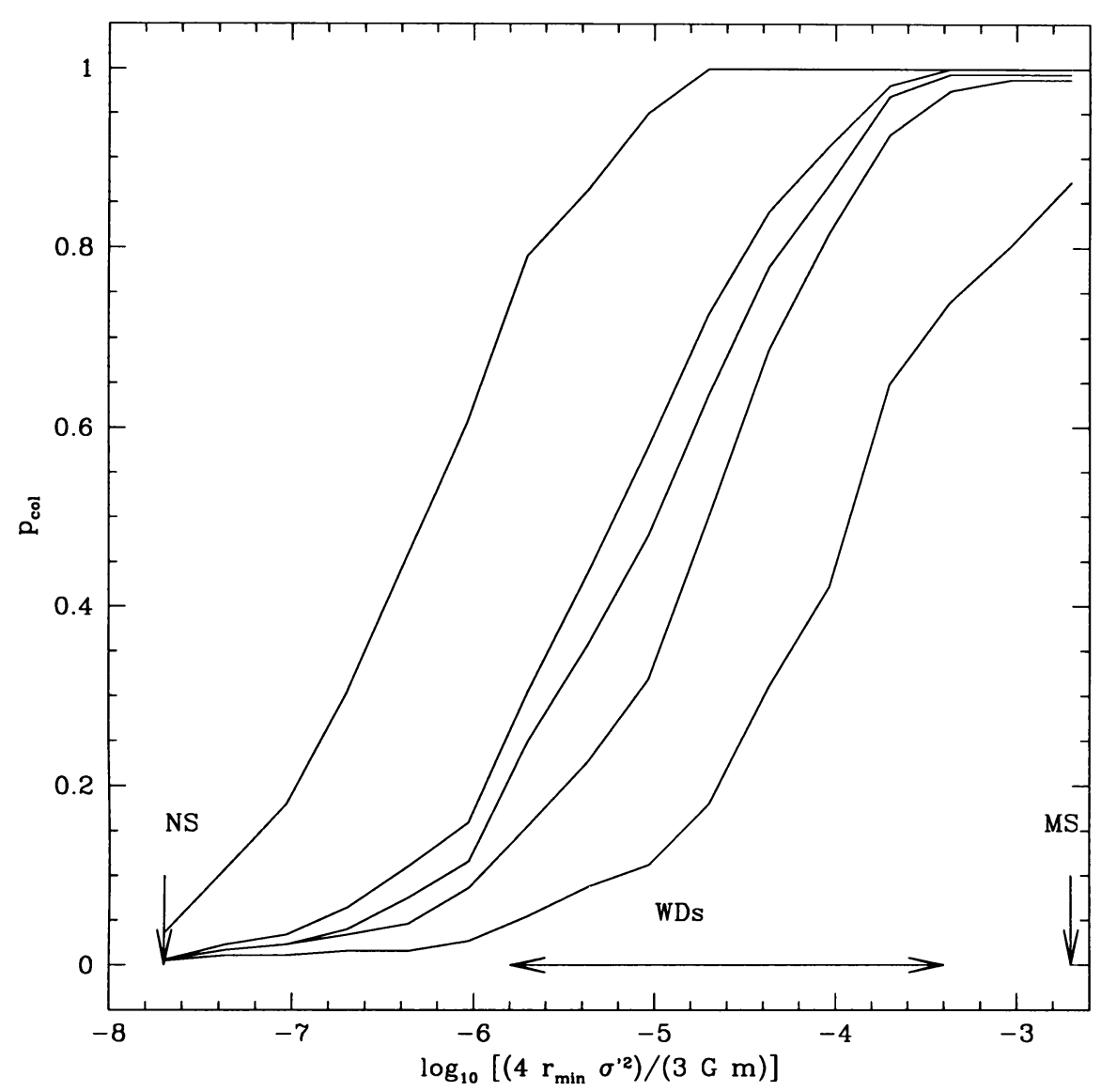

Figure 3. The probability of collision of an immortal binary as a function of the physical distance of separation $r_{\min }$ that yields a collision. The arrows mark the location of neutron stars (NS), white dwarfs (WDs) and main sequence stars (MS) for fiducial parameters (see text). The multiple lines correspond to different depths of the central potential $\Phi(0) / \sigma_{s}^{2}=2,6,11,14$ and 128 (right to left).

A detailed analysis of the factors that lead to the remarkably small distances of closest approach shows that three independent physical effects contribute. First, as is well-known, resonant encounters increase the collision probability as the three stars form a long-lived interacting three-body system. The cross section for two stars to pass within $0.01 a$ when a hard, circular binary interacts with a third star is of order $a^{2}$ times the gravitational focusing term. Hence, strong encounters immediately imply elevated 
collisional cross sections. Cross section for close encounters have been derived by Hut and Inagaki (1985) and Sigurdsson and Phinney (1993); our own calculations of these cross sections are in good agreement with the previous studies.

Second, 3B binary hardening involves a large number of successive encounters. The repetitive nature raises the probability that at least one collision will occur as the binary ages (and is analogous to the effect of resonant interactions on collision probability). Although only a fraction of the simulated encounters with pericenter less than $\zeta a$ are strong (roughly $3 / \zeta$ ), these significantly increase the cumulative probability of a collision for a binary of a given hardness.

Third, the value of the binary eccentricity appears to play some role, albeit a subdominant one, in enhancing collisions. The cross section for close encounters depends upon $e$ : the probability for an $e=0.7(0.99) \mathrm{bi}$ nary to suffer a collision is about 1.6 (10) times that of a circular binary. The $3 \mathrm{~B}$ binaries have a nearly thermal eccentricity distribution and this raises the collision probability over estimates made based on purely circular calculations. A related but distinct physical effect is that most of the simulated encounters are weak, angular momentum changing events. Thus, the binary eccentricity undergoes some diffusion in the interval between the strong, resonant encounters. This diffusion provides a small enhancement of the collision probability because the binary explores some very eccentric configurations.

Taken together, these factors appear to account for the high propensity for collisions to occur during the hardening process.

\section{Effective Heating}

When collisions occur the net heat extracted from an immortal binary is altered. Figure 4 illustrates the average change in binary binding energy per immortal binary until ejection or collision. All subsequent consequences of the collision have been ignored in so far as the heating is concerned (i.e. changes due to altered stellar evolution, mass ejected to infinity by the collision, etc.). Also, no attempt has been made to differentiate the cluster heating via bound versus ejected single stars; the inferred heating is simply the average change in binary binding energy. The total heating (solid line) decreases as the finite size of the objects and the collision probability increases. Two contributions to the total are independently noted: the average heating by binaries ultimately ejected and by binaries that collide. The results are presented to allow one to estimate easily the steady-state heating rate as the generation rate of immortal binaries $\left(\dot{n}=0.75 n^{3} G^{5} m^{5} / \sigma_{s}^{9}\right.$ GH93) times the mean heating per binary $(d E / d t=\dot{n} \overline{\Delta E})$. 


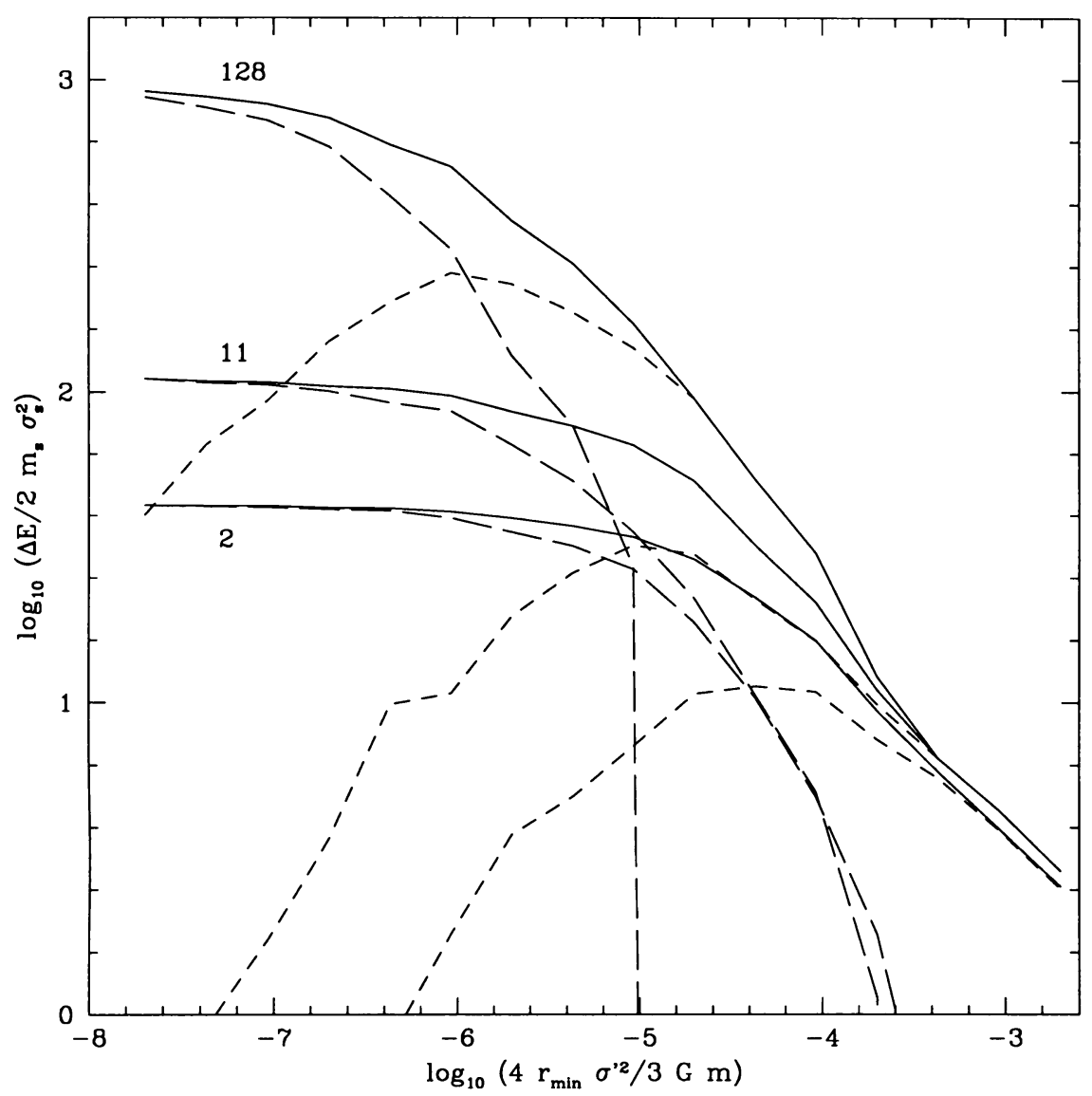

Figure 4. The change in binding energy per immortal binary is given as a function of the physical distance that yields a collision. The solid lines are the total heating; the heating of ejected binaries (long dashes) and of binaries that collide (short dashes) are independently noted. Groups of three lines are marked by the depth of the potential in units of $\Phi(0) / \sigma_{s}^{2}$.

\section{Conclusions}

The steady-state distribution of binding energy, eccentricity, distance of closest approach, and maximum recoil velocity for $3 \mathrm{~B}$ binaries has been calculated. This generalizes previous calculations of the distribution of binding energy for hardening binaries (Goodman and Hut, 1993) and estimates of the collisional probability based on averaged cross sections. The collision 
probability (meaning the probability that two point masses pass within a distance of two physical radii) is significant for objects as small as white dwarfs in typical cluster potentials with typical velocity dispersions. The heating efficiency is diminished when collisions are included. When 3B binaries are responsible for heating post collapse cores the results provide a direct relationship between the stellar collision rate, the stellar heating rate and the rate of energy transport through the cluster as a whole.

\section{Acknowledgements}

We acknowledge support from the IAU Symposium organizers, NASA NAGW2224, NSF AST-86-57467 and AST-91-19475.

\section{References}

Davies, M.B., Benz W. and Hills J.G. (1994), Ap. J. 424, 870.

Davies, M.B., Benz W. and Hills J.G. (1993), Ap. J. 411, 285.

Goodman, J. and Hut, P. (1993), Ap. J. 403, 271.

Heggie, D. C. and Hut, P. (1993), Ap. J. Supp. 85, 347.

Hut, P. (1985) in Dynamics of Star Clusters, IAU Symposium No. 113, eds. J. Goodman and P. Hut, Reidel, Dordrecht, p. 231.

Hut, P. and Inagaki, S. (1985), Ap. J., 298, 502.

Goodman, J. (1989) in Dynamics of Dense Stellar Systems, ed. D. Merritt, Cambridge University Press, New York, p. 183.

Kochanek, C.S. (1992), Ap. J., 385, 604.

Mardling, R. A. (1995a) Ap. J. 450, 722.

Mardling, R. A. (1995b) Ap. J. 450, 732.

Sigurdsson, S. and Phinney, E. S. (1993), Ap. J., 415, 613.

Takahashi, K. and Inagaki, S. (1991), P. A. S. J., 43, 589. 